picks up the activities of the monomer building blocks as well as the binary combinations.

\section{High-content screening}

The amount of information that can be gleaned from a screen can be increased by using cell-based systems. Screens such as those offered by Amersham Biosciences, Evotec OAI in Hamburg, Germany, and Vertex Pharmaceuticals in San Diego, California, allow complex biological data on lead-compound behaviour to be collected.

"Although the industry has been doing in vitro assays for a long time, there is a big increase in complexity when you start thinking about using whole cells," says John Anson, vice-president of systems development at Amersham. For instance, instead of just measuring the binding of a ligand to a receptor in vitro, you might now need to track the movement of a labelled molecule from the cytoplasm to the nucleus. Researchers are also beginning to measure more than one event at a time, for instance by using two different reporter molecules, and this is adding to the complexity.

The increased intricacy of assay systems is changing perceptions of the screening process. "The ability to track the internalization or translocation of a cellular component allows you to think more deeply about what you want to get out of a screen," says Paul Negulescu, vice- president of discovery biology at Vertex.

Although most researchers would admit that a degree of serendipity operates in screening for hits and leads, most screens are hypothesis-driven, using assays designed to test the effects of compounds on a particular protein target. But CombinatoRx, a two-year-old company based in Cambridge, Massachusetts, has taken a very different approach. It screens binary combinations of existing drugs to see whether drugs that have known effects when acting singly might have different, unexpected, effects when used in combination.

\section{Double value}

Most drugs do not, in fact, target single proteins, explains the company's chief executive, Alexis Borisy. Instead they interact with a number of targets at a variety of potencies.

"Recognizing the inherent complexity of biological systems, we want drugs that will interact with multiple points in a pathway, rather than the 'sledgehammer' strategy of affecting just one key protein," he says, reversing the usual mantra that drugs should be as selective as possible.

The data generated by CombinatoRx's screens are built into 'interaction spaces' to illustrate the dose-response relationship of the two drugs in combination. At present, the company has
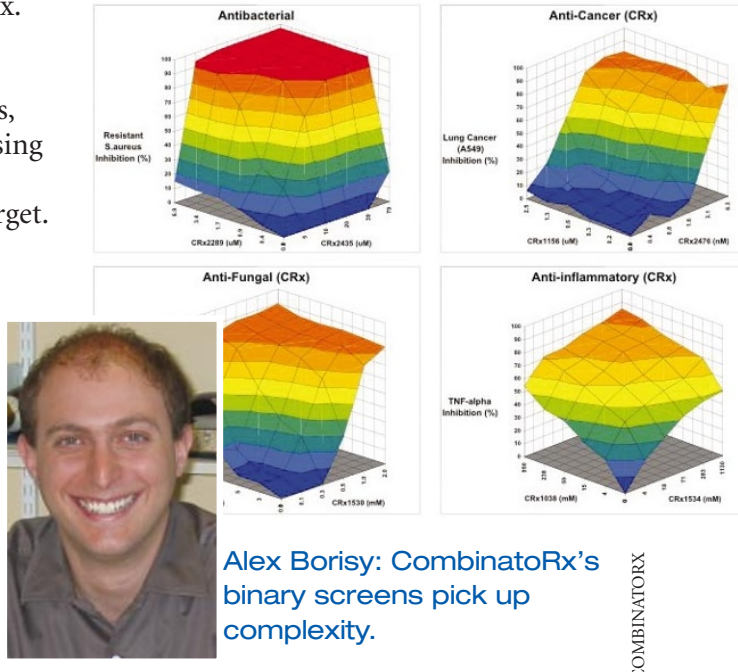

Alex Borisy: CombinatoRx's binary screens pick up complexity.

a screening library of 12.5 million binary combinations. And because all these molecules have already been approved by the US Food and Drug Administration, and are mostly off-patent, it should be possible rapidly to develop any hits for further testing. CombinatoRx plans to start clinical trials on its first sets of binary combinations later this year.

For all these new approaches to screening, the number of new compounds entering clinical trials in the coming years will be the ultimate measure of their success.

Adam Smith is editor of Nature Reviews Drug Discovery.

\title{
FRAGMENTING THE PROBLEM
}

Bioassay-based screening approaches search for hits with, for example, inhibitor constants at least in the low micromolar range. To get this much potency, a minimum number of interaction points between the molecule and the target protein are needed, which means that only larger compounds generate an initial hit.

Over the years, the compounds held by drug-discovery companies in their collections have been getting bigger, as screeners and medicinal chemists have chased the goal of potency. But this brings its own problems. "It is generally accepted that

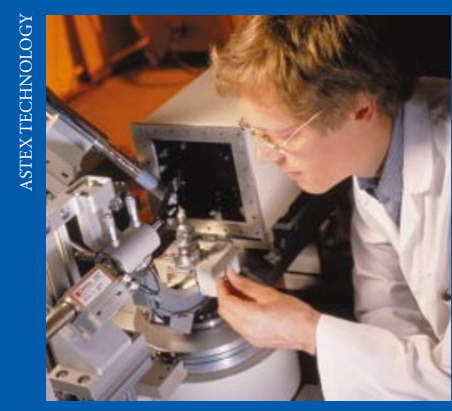

Astex is building leads with X-ray crystallography. larger compounds lead to more late-stage attrition," explains Harren Jhoti, cofounder and chief scientific officer of Astex Technology, a lead-discovery company based in Cambridge, UK. "Chemically refining large initial hits to work out which groups are important or not can be very time consuming." The highly sensitive screening techniques that are now emerging, such as nuclear magnetic resonance and surface plasmon resonance, are providing the opportunity to search for lower-affinity hits, in the high micromolar or even low millimolar range. This opens up the possibility of screening for smaller starting compounds 'chemical fragments' - which can then be built up into druglike molecules by adding optimal functionalities, ideally using the structure of the target protein derived from X-ray crystallographic data. This, argues Jhoti, should allow for "a more directed path from hit to the lead than the hit-and-miss process of refining larger molecules". Astex now has libraries of chemical fragments that bind in the low millimolar range, and already has examples of nanomolar lead compounds that were generated from millimolar hits.

Another company working with fragments is Graffinity Pharmaceuticals, in Heidelberg, Germany, which is taking advantage of the sensitivity of its optically based screening platform to test microarrays of chemical fragments. Technological challenges aside, another barrier to the development of fragment-based approaches is the generally held perception that high potency in binding to a target is a prerequisite for further development of a compound.

"There is a change of mindset needed in order to convince a chemist that a millimolar hit is going to be of use," says Jhoti. 